\title{
Research of Preschool Teacher Competency Model Construction and Its Evaluation Under Two-Child Policy
}

\author{
Yunyan Liang ${ }^{1}$ \\ ${ }^{1}$ Shunde Polytechnic, Foshan, Guangdong, 528300 \\ 346591653@163.com
}

\section{KEYWORDS: Two-Child Policy; Preschool Teacher; Competency Model}

\begin{abstract}
This study was designed to investigate the preschool teachers who should have professional competency structure ability. In this paper, behavioral event interviews and questionnaires, competency from the perspective of preschool teacher competency model were studied.
\end{abstract}

\section{Introduction}

Education is the foundation; education plans, teacher-centered. Teacher quality directly affects the quality of education, thus affecting the cause of China's modernization Health Promotion. Therefore, the state and society increasingly high quality requirements for teachers, research on teacher competency more and more attention. Competency (Competency) refers to a work can outstanding performance and average performers distinguish individual potential, deep features, it includes five dimensions: knowledge, skills, self-concept, character and motivation. The Competency Model (Competency Model) refers to the specific job requirements of combining a set of competency, reflecting effectively complete knowledge, skills and qualities required for a given job a unique combination. Competency model is the logical starting point and the cornerstone of modern human resource management system, which provides a theoretical basis for the selection of personnel, training, performance evaluation and career planning, plays a fundamental role in human resource management decisions activities.

Research on teacher competency model can be traced back to the 20th century, the ability - based Teacher Education (CBTE) and people of this teacher education (HBTE) movement, ability-based teacher education emphasizes skills (or knowledge) culture, and people of the teacher education directly concern individuals themselves (or quality). Affected CBTE and HBTE movement teacher competency research increasingly become a hot topic of educational research. Where a representative of "a high-performance model of teachers' report Hayi (Hay McBer) submitted to the US Department of Education and Employment, the report proposes that contains five kinds of competency group, 15 competency teacher competency model. There are also researchers through questionnaires, found that teacher competency include the learning environment, teaching basic ability to cooperate teachers' professional commitment, discipline, teachers, teacher reflection, teacher, effectiveness and leadership, and that this eight factors can be classified as two categories, namely education competency (Educative competence) and Collaboration competency (Collaborative Competence). Teachers Denison (Danielson), who proposed competency model 
contains four dimensions, namely planning and preparation, classroom environment monitoring, teaching and professional responsibility.

\section{Overview of Competency}

After the concept of competency proposed, many researchers have put forward their own competency views. McClelland (1973) that the competency "direct the performance of similar work or live in or linked to other important achievements of knowledge, skills, abilities, traits or motives." Boyatizis $(1982,1994)$ the competency defined as "a person having any qualities and is used to produce a successful performance in the role of a life, the potential characteristics of this individual, may be the motivation, traits, skills, self-image or social role, or knowledge. " Spencer (1993) proposed competency means "capable of a work (or organization, culture) in those who are high achievers and average performers distinguish individual features to potential, he can be a motivation, character, self-image, attitude or values, certain areas of knowledge, cognitive or behavioral skills - anything that can be reliably measured or counted and could significantly distinguish the individual and the general performance of outstanding features" Helley (2001) that "competency is generally defined as a feature, this feature allows a person in a productive way to complete his or her work, and that this feature can be based on an acceptable performance criteria to measure It contains many aspects of "knowledge, skills, abilities, traits, attitudes, motivation and other acts.

Since competency is still not a let people accepted the concept, but from can also see some consensus: the first competency is comprehensive, the competence is constituted by a variety of factors, it is the combination of external knowledge and skills and internal social role, self concept and motivation factors. Secondly competency with recognition, not all of the knowledge, skills, social role, motivation belong to the category of competency, only can distinguish between high performance and general performance of the ability to competency. Third competency is dynamic, competency can learn and transfer, can be improved through training, etc.. The final competency is closely related to the work, only the ability of individuals to use their own to work in order to be called the competency.

\section{Construction Method of Competency Model}

The main way to identify competency is to establish competency model. Spencer et al., Based on years of research, the development of a more comprehensive behavioral event interview procedures and coding methods. Specifically, the use of behavioral event interview build competency model, generally through the five basic steps: First, determine the performance standards. Ideal performance standard is "hard" indicators, for teachers, "hard" indicators include whether the honorary title of teacher, and published articles, teaching quality, etc., or you can take so that principals, colleagues, parents and students nominated method. Next, choose criterion sample, choose the excellent group and general group has determined based on performance criteria. Again, the sample data obtained criterion relating to competency. Currently the most important method used is the behavioral event interview. Fourth, analyze data and build competency model. Analyzed by the obtained data, identify competency can distinguish outstanding and ordinary persons. Finally, verify competency model. Usually three methods to validate the competency model: (1) investigation "cross validity." Re-select the second sample, again using behavioral event interview to collect data, competency model has been established to study other samples if applicable. (2) investigation "construct validity." According to the preparation of the competency model to other 
forms of evaluation tools, select a different sample Surveying investigated with outstanding performance and the general who on competency model has been established in the key competencies Is there a significant difference in the results of the evaluation. (3) Investigation "predictive validity." Using behavioral event interview or other selection or training quiz, and then track examine whether they behave better work in the future

\section{Reliability and Validity Study}

To test the performance of blue-chip group and group differences on each level of competency that whether caused by the length of the interview, we first of two interviews of words and interview time homogeneity of variance test. The results showed a significant difference, the blue-chip group in time for the interview and the interview length was higher than performance level group, but this difference was not statistically significant $(\mathrm{P}>0.05)$. Visible, it will not affect the length of the interview group differences merit and performance level group on competency. Secondly, tentatively out of the 22 competencies of frequency, related to average grade score and the highest grade scores and interviews length between analysis found were only three, one and two and interviews related to the length, indicates that three Indicators are more stable, less susceptible to influence the length of the interview, relatively speaking, the average grade scores have better stability and distinguishability, which past results are consistent with similar studies. [12] Third, in the present study, the two coders on 14 subjects code classification consistency coefficient from 0.559 to 0.750 , the overall classification consistency coefficient of 0.645 ; coding reliability coefficient from 0.717 to 0.850 overall reliability coefficient of 0.784 . It shows higher coding consistency between the two coders. Fourth, calculate two coders coded for each subject frequency, average rating scores, the highest level score Pearson correlation coefficient used to further investigate the consistency between the two coders. The results showed that the two coders on 22 competency code, were only 4, 4, 3 competency items on frequency, the average grade score, the highest score of the three indicators rank correlation was not significant, The vast majority showed a significant correlation. Indicating a higher two coders coding consistency, in line with psychometric laid a reliable foundation for the subsequent data analysis and model extraction.

Competency average grade score, score the highest level and frequency difference between the two coders calculate average test scores for a competency rating scores coding, standardization after conversion, the test groups were tested an average rating for each competency the difference between scores. In the 22 competencies, the two groups in achievement motivation, career preferences, teamwork, developing others, differences in professional knowledge and skills, learning ability, sense of service and influence among these eight characteristics statistically significant, other features the difference was not statistically significant, but the performance level was significantly higher than the blue-chip group. $t$ test results also show that the sum of the blue-chip average grade score was significantly higher than the sum of the average grade score performance level group, the difference was significant statistical significance. Highest rating score normalized t test results showed that the highest level of two points in achievement motivation, career preferences, developing others, there are significant differences between the learning ability, service awareness and influence of these six competencies. At the same time, the sum of the highest merit rating score was significantly higher than the sum of the highest performance rating score level group, the differences were statistically significant $(t=5.364, \mathrm{P}=0.000)$. Merit and performance level group set competency frequency of occurrence summary comparison shows that the frequency difference on the two groups occurred in the development of others $(\mathrm{t}=2.238, \mathrm{P}=0.044)$ and influence ( $\mathrm{t}=2.191, \mathrm{P}=0.049$ ) These two competency with statistical significance, but the blue-chip 
group competency sum frequency of occurrence and competent performance level group difference was statistically significant sum frequency characteristics occur with significant $(t=4.697, P=0.001)$. Considering the above differences in test results can be drawn as early as eight teachers to identify competencies: professional knowledge and skills, developing others, service awareness, learning ability, achievement motivation, career preferences, influence, and teamwork.

Competency Checklist statistical results in pre-revised "competency dictionary" basis, the researchers developed a "teacher early Competency Checklist" and then the main class teacher and worked as teachers and teaching master classes conducted director investigation, let them elect master classes for nursery teachers are the most important items 10-12 from 22 competencies in competency. 120 questionnaires were distributed and recovered 105 questionnaires, 102 valid questionnaires. The recovered Checklist result statistics, frequency according to the competency of the sort occurs, as shown in Table 2, the top 12 of the competency are teamwork, learning ability, professional knowledge and skills, service awareness, adaptability, career preferences, honesty, integrity, team management, innovation, problem-solving ability, influence and relationship building.

\section{Conclusion}

Early language teacher competency model includes specialized knowledge, language skills, lifelong learning, responsibility, good teacher-student relationship, organization and management skills, language teaching ability, enthusiasm, initiative, reading skills, teamwork, personal literary accomplishment, humor sexual, psychological and pedagogical knowledge, communication skills, the ability to self-reflection, tolerance, quality, problem solving, listening skills and flexibility of 21 competencies, and this 21 is divided into six dimensions competency, followed service awareness, achievement motivation, professional knowledge, professional skills, relationship skills and personal qualities.

\section{REFERENCE:}

[1]Spencer L M, Spencer SM. Competence At Work: Models for Superior Performance. New York: John Wiley \& Sons ,Inc.,1993.

[2]Williams R S. Performance Management. London: International Thomson Business Press, 1998.

[3]Bisschoff, Bennie Grobler. The Management of Teacher Competence. Journal of In Service Education, 1998, (24).

[4]Danielson, Charlotte. Enhancing Professional Practice: A Framework for Teaching. Alexandria, VA: Association for Supervision and Curriculum Development, 1996. 